\title{
Reac Technology and Veterinary Medicine: A Horizon of Opportunities
}

\author{
Eraldo Sanna Passino ${ }^{1 *}$, Salvatore Rinaldi ${ }^{2}$ \\ ${ }^{1}$ Department of Veterinary Medicine and Comparative Surgery Center, Ospedale Veterinary Teaching Hospital, University of Sassari \\ ${ }^{2}$ Istituto Rinaldi Fontani, Florence
}

Received: 06 December, 2016; Accepted: 12 December, 2016; Published: 21 December, 2016

*Corresponding author: Eraldo Sanna Passino, Department of Veterinary Medicine, University of Sassari, Via Vienna, 2. 07100 Sassari (Italy), Tel. +39 229403 - 229471, mobile +39329 3808644, fax+39079 229408; E-mail: esp@uniss.it

\section{Abstract}

The authors describe the principles of the physical and biological functioning of REAC and suggest its use in veterinary clinical practice.

Radio Electric Asymmetric Conveyer (REAC) Technology is a platform technology for bio and neuro modulation, patented at international level (WO2001EP07800, EP1301241, US7333859 and other patents pending).

The scientific background of REAC technology platform is based on a fundamental phenomenon for life: cells have an asymmetric structure, as several cellular components, including plasma membrane, cytoskeleton or organelles, asymmetrically organized. This cell structure implies that ion channels are asymmetrically distributed throughout cellular membrane and this generates ionic fluxes. These ionic fluxes in turn produce currents and these currents produce bioelectric fields. This phenomenon takes the name of "Cell polarity".

Cell polarity is at the base of correct cell bioelectric activity and is implicated in important processes that influence normal cell functions such as cell differentiation, proliferation, morphogenesis, migration and neurotransmission in unicellular and multicellular organisms. The establishment and maintenance of cell polarity involves many processes including signaling cascades, membrane trafficking events and cytoskeletal dynamics, all of which need to be coordinated in a highly regulated manner. Epigenetic factors, trauma, infections and other factors alter the mechanism of action of the cell polarity. This blocks the correct production of ion fluxes in cells and tissues, and consequently it alters electro-metabolic activity, functions and reparative processes in cells and tissues. Dysregulation of cell polarity can cause developmental disorders, diseases and cancer.

The mechanism of action of REAC Technology is aimed to restore the correct cell polarity. In fact, REAC technology is able to properly asymmetrically convey the ion fluxes and consequently the ion currents, "re-setting" the altered bioelectric fields and therefore recovering the correct cell electro-metabolic activity.

This optimization of cell polarity allows the proper recovery of cellular electrometabolic processes, moreover cell migration, proliferation and differentiation, by promoting reparative and regenerative process of tissues.

REAC technology is so effective to induce remarkable biological effects at many scales, from gene modulations up to functional neuro biological optimization.

The results obtained in vitro and in vivo suggest a role of REAC in regenerative medicine that opens new scenarios in neurological, vascular, orthopaedic trauma and tissue repair.

The effects of REAC Technology are completely pervasive and have no limit in depth in the tissues to be treated.

The key features of REAC technology are the absolute safety, ease of use, the presence of effective standardized protocols validated by years of use and the ability to be associated with other pharmacological and surgical treatments, supporting and enhancing them.

All these features can open up new perspectives of REAC technology use in veterinary medicine.

\section{References}

1. http://www.irf.it/2016/it/pubblicazioni.html 